\title{
The incidence of postoperative hemorrhage after tooth extraction in patients with thrombocytopenia
}

\author{
Sang-Hoon Kang, Min-Jun Kang \\ Department of Oral and Maxillofacial Surgery, National Health Insurance Service Ilsan Hospital, Goyang, Korea
}

\begin{abstract}
J Korean Assoc Oral Maxillofac Surg 2021;47:190-196)
Objectives: The risk of bleeding after tooth extraction in thrombocytopenia patients remains unclear. Therefore, the present study aimed to assess the risk of bleeding after tooth extraction in patients with thrombocytopenia.

Materials and Methods: The study included 220 patients who had a medical history of thrombocytopenia and underwent tooth extraction (330 teeth). The patients were divided into those who had thrombocytopenia (platelet count $<150 \mathrm{k}$ ) immediately before the tooth extraction, and those who had platelet counts that were between 150k and 250k before the extraction. Bleeding complications were recorded and compared between the patient groups.

Results: Of the 220 patients, 130 underwent tooth extractions while having thrombocytopenia (platelet count $<150 \mathrm{k}$ ), and 90 had platelet counts that were between $150 \mathrm{k}$ and $250 \mathrm{k}$ before tooth extractions. Bleeding complications occurred in 11 patients $(5.0 \%)$ of the 220 patients. Among those 11 patients with bleeding complications, 10 patients $(7.7 \%)$ had thrombocytopenia (platelet count $<150 \mathrm{k})$ of the 130 patients, and 1 patient $(1.1 \%)$ had a normal platelet count of the 90 patients. There was a significant difference between the patient groups regarding bleeding after extractions $(P<0.001)$. No significant difference in the incidence of post-extraction bleeding was found between the subgroups by platelet count within the thrombocytopenia group.

Conclusion: Thrombocytopenia (platelet count $<150 \mathrm{k}$ ) increases the risk of post-tooth extraction bleeding. Therefore, bleeding control under the proper evaluation of hemostasis and performing delicate tooth extraction procedures using hemostatic plugs is necessary during the tooth extraction of patients with thrombocytopenia.
\end{abstract}

Key words: Thrombocytopenia, Postoperative hemorrhage, Tooth extraction, Platelet count

[paper submitted 2020. 12. 31 / revised 2021. 2. 9 / accepted 2021. 2. 17]

\section{Introduction}

Some patients with thrombocytopenia experience bleeding after an invasive dental procedure. For tooth extractions, which are common dental procedures, predicting and preventing postoperative hemorrhagic complications is important for patients with thrombocytopenia, who are affected by hemostatic measures.

Studies have reported that the daily use of aspirin, which

\footnotetext{
Sang-Hoon Kang

Department of Oral and Maxillofacial Surgery, National Health Insurance Service Ilsan Hospital, 100, Ilsan-ro, Ilsandong-gu, Goyang 10444, Korea TEL: +82-31-900-0267

E-mail:omskang@nhimc.or.kr

ORCID: https://orcid.org/0000-0003-3335-3040

(c) This is an open-access article distributed under the terms of the Creative Commons Attribution Non-Commercial License (http://creativecommons.org/ licenses/by-nc/4.0/), which permits unrestricted non-commercial use, distribution, and reproduction in any medium, provided the original work is properly cited.

Copyright (C) 2021 The Korean Association of Oral and Maxillofacial Surgeons. All rights reserved.
}

affects platelet function, does not cause serious hemorrhagic complications after tooth extraction ${ }^{1-3}$. Patients who take aspirin rarely cease their aspirin use before tooth extraction. Local hemostatic measures are recommended over the cessation of aspirin (anti-platelet) administration before tooth extraction ${ }^{1,3}$.

However, it remains unclear about whether there is a high incidence of hemorrhagic complications after tooth extraction in patients with thrombocytopenia who do not have a reduced platelet function, but a reduced platelet count ${ }^{4}$. Tooth extraction may be performed on a patient with thrombocytopenia whose platelet count has not increased even after platelet transfusion. In such cases, the patient and the surgeon must bear the risk of postoperative bleeding and understand the magnitude of the risk.

In this study, we examined the association between thrombocytopenia and hemorrhagic complications after tooth extraction to investigate the risk of bleeding in patients with thrombocytopenia following tooth extraction. 


\section{Materials and Methods}

The medical records of patients who had been diagnosed with thrombocytopenia and who underwent tooth extractions between 2001 and 2019 at our hospital were reviewed. Patients were included if they satisfied the following criteria: 1) had a medical history diagnosed with thrombocytopenia at our institution and had a clear medication history and tooth extraction records; 2) had not undergone radiation therapy, tumor surgery, or osteomyelitis treatment in the jawbone area; and 3) their platelet count was determined through a blood test before tooth extraction. Patients were excluded if they had undergone tooth extractions before the onset of thrombocytopenia or had unclear blood test results or test dates that were diagnosed at another hospital and later referred to our hospital.

Two hundred and twenty patients who satisfied all of the aforementioned criteria were included. Patients who were diagnosed with thrombocytopenia, but had a normal platelet count at the time of tooth extraction were assigned to the control group. These patients had platelet counts $>150 \mathrm{k}$ and $<250 \mathrm{k}$. A patient was deemed to have thrombocytopenia at the time of tooth extraction if the platelet count was $<150 \mathrm{k}$ at the time of the procedure. Patients with thrombocytopenia were assigned to the experimental group. A total of $330 \mathrm{ex}-$ traction sites were examined.

\section{Extraction techniques and hemorrhagic complications after tooth extraction}

The patients were referred to the Department of Oral and Maxillofacial Surgery for tooth extraction. The indications for tooth extraction included the presence of remnant tooth roots, severe dental caries making the tooth non-restorable, tooth fractures, severe periodontal inflammation, and pericoronitis. In the case of severe signs of infection, tooth extractions were performed after the infections were relieved with antibiotic therapy.

The methods of tooth extraction included extractions with sutures and surgical extractions involving an alveolar osteotomy and root splitting. Patients took orally prophylactic antibiotics before the tooth extraction. Patients were informed to bite on wet gauze and return home after the confirmation of hemostasis. An analgesic was prescribed to all the patients before extraction (ibuprofen $300 \mathrm{mg}$ or acetaminophen 300 $\mathrm{mg}$ ) and after extraction (ibuprofen $300 \mathrm{mg}$ or acetaminophen $300 \mathrm{mg}$ for 3-7 days). Antibiotics were also administered after tooth extraction depending on whether or not postoperative infection occurred.

Patients with platelet count $\geq 150 \mathrm{k}$ underwent tooth extractions without consultations at the internal medicine department even though they had previously been diagnosed with thrombocytopenia. Patients with platelet count $<150 \mathrm{k}$ underwent tooth extractions after consultations at the internal medicine department. A decision was made on whether to perform the extraction after platelet administration or after hospitalization based on a response from the internal medicine department that examined the patient. All the patients were informed of the possibility of hemorrhagic complications after tooth extractions and were discharged once hemostasis was achieved. They were told to return to the hospital if post-extraction bleeding occurred.

For patients who had hemorrhagic complications after tooth extraction, the first choice for bleeding control was biting wet gauze to achieve hemostasis by compressing the bleeding site of the tooth extraction area. If needed, a collagen or gelatin sponge was placed in the extraction socket to achieve hemostasis through compression. Hemostasis was also achieved through suturing the adjacent areas. Patients were discharged after hemostasis was confirmed.

\section{Statistical analysis}

The thrombocytopenia group consisted of patients with platelet count $<150 \mathrm{k}$ with the control group of patients with platelet counts between $150 \mathrm{k}$ and $250 \mathrm{k}$. The Student $t$-test was used to examine the differences between the two groups according to age and platelet counts. The chi-square test was used to examine the differences in gender, the number of patients who had post-extraction bleeding complications, the amount of platelet transfusions received before tooth extraction, and the extraction techniques including alveolar osteotomies between the group that underwent tooth extractions with thrombocytopenia and the control group with normal platelet counts.

Patients with thrombocytopenia were divided into three groups according to their platelet counts: $<50 \mathrm{k},>50 \mathrm{k}$ and $<100 \mathrm{k}$, and $>100 \mathrm{k}$ and $<150 \mathrm{k}$. Statistical analysis for these subgroups was performed with the same variables. Additionally, the number of teeth extracted and the frequency of bleeding was investigated for different extraction sites for each group. The extraction sites involved the maxillary and mandibular regions, which were further divided into anterior, premolar, and molar regions. In cases where a simple extrac- 
tion and sutures were performed in the maxilla or mandible, whether or not surgical extractions involving alveolar osteotomies were performed was investigated. Statistical analysis was performed using IBM SPSS Statistics (ver. 23.0; IBM, Armonk, NY, USA). A $P$-value $<0.05$ was considered statistically significant.

\section{Results}

A total of 220 patients were examined - 130 were male and 90 were female. Tooth extractions were performed in 130 patients with thrombocytopenia where the platelet counts were less than $150 \mathrm{k}$. Ninety patients with platelet counts between $150 \mathrm{k}$ and $250 \mathrm{k}$ within the normal limit underwent tooth extractions.

Three hundred and thirty teeth were extracted from a total of 220 patients. One hundred and sixty-two teeth were extracted from the maxilla, and 168 teeth were extracted from the mandible. Sixty anterior teeth, 87 canines and premolars, and 183 molars were extracted. Forty-four extractions were surgical extractions accompanied by alveolar osteotomies.

Eleven $(5.0 \%)$ of the 220 patients exhibited postoperative bleeding complications after the tooth extractions. Of the 130 patients who underwent tooth extractions in a thrombocyto- penic state, 10 patients $(7.7 \%)$ had postoperative hemorrhagic complications after the procedures. Of the 90 patients with normal platelet counts, one patient $(1.1 \%)$ had a postoperative hemorrhagic complication after tooth extraction. There was a significant difference in the number of patients who had postextraction bleeding between the two groups $(P=0.030)$.(Table 1) One of the 44 patients who underwent tooth extractions involving alveolar osteotomies had a post-extraction hemorrhagic complication. Of the 11 patients who had post-extraction hemorrhagic complications, four had platelet counts $<50 \mathrm{k}$, three had platelet counts $>50 \mathrm{k}$ and $<100 \mathrm{k}$, and three had platelet counts $>100 \mathrm{k}$ and $<150 \mathrm{k}$.(Table 2) No significant difference in the incidence of post-extraction bleeding was found between the subgroups by platelet count within the thrombocytopenia group $(P=0.293)$.

Of the 130 patients who underwent tooth extractions with thrombocytopenia, 18 patients $(13.8 \%)$ received a platelet transfusion before the procedure. None of the 90 patients who underwent tooth extractions with a normal platelet count received a platelet transfusion before the procedure $(P<0.001)$. One of the patients who had a hemorrhagic complication had received a platelet transfusion before the tooth extraction. Of the 27 patients who underwent tooth extractions with platelet counts $<50 \mathrm{k}$ in the thrombocytopenia group, 14 patients

Table 1. Characteristics and incidence of post-extraction bleeding according to the preoperative PLT

\begin{tabular}{|c|c|c|c|}
\hline \multirow{2}{*}{ Variable } & \multicolumn{2}{|c|}{ Groups according to the preoperative PLT $(n=220)$} & \multirow{2}{*}{$P$-value } \\
\hline & PLT<150k $(n=130)$ & $150 \mathrm{k}<\mathrm{PLT}<250 \mathrm{k}(\mathrm{n}=90)$ & \\
\hline Sex (male:female) & 79:51 & $51: 39$ & 0.578 \\
\hline Age $(y r)$ & $63.1 \pm 15.1$ & $65.7 \pm 13.7$ & 0.192 \\
\hline PLT & $83.6 \mathrm{k} \pm 38.1 \mathrm{k}$ & $209.4 \mathrm{k} \pm 42.0 \mathrm{k}$ & $<0.001 *$ \\
\hline Post-extraction bleeding patient & $10(7.7)$ & $1(1.1)$ & $0.030 *$ \\
\hline Transfusion patient before tooth extraction & $18(13.8)$ & $0(0)$ & $<0.001 *$ \\
\hline Surgical extraction with osteotomy & $29(22.3)$ & $15(16.7)$ & 0.392 \\
\hline
\end{tabular}

(PLT: platelet count, k: 1,000 unit)

$* P<0.05$.

Values are presented as number only, mean \pm standard deviation, or number $(\%)$.

Sang-Hoon Kang et al: The incidence of postoperative hemorrhage after tooth extraction in patients with thrombocytopenia. J Korean Assoc Oral Maxillofac Surg 2021

Table 2. Characteristics and incidence of post-extraction bleeding according to preoperative PLTs within the thrombocytopenia patient group (PLT<150K)

\begin{tabular}{|c|c|c|c|c|}
\hline \multirow{2}{*}{ Variable } & \multicolumn{3}{|c|}{ Subgroups within the thrombocytopenia group $(n=130)$} & \multirow{2}{*}{$P$-value } \\
\hline & $<50 \mathrm{k}(\mathrm{n}=27)$ & $50 \mathrm{k}-100 \mathrm{k}(\mathrm{n}=55)$ & $100 k-150 k(n=48)$ & \\
\hline Sex (male:female) & $18: 9$ & $30: 25$ & $31: 17$ & 0.454 \\
\hline Age (yr) & $65.0 \pm 17.0$ & $60.3 \pm 15.4$ & $65.2 \pm 13.4$ & 0.202 \\
\hline PLT & $27.8 \mathrm{k} \pm 10.9 \mathrm{k}$ & $76.3 \mathrm{k} \pm 14.3 \mathrm{k}$ & $123.4 \mathrm{k} \pm 14.9 \mathrm{k}$ & $<0.001 *$ \\
\hline Post-extraction bleeding patient & $4(14.8)$ & $3(5.5)$ & $3(6.3)$ & 0.293 \\
\hline Preoperative transfusion patient & $14(51.9)$ & $4(7.3)$ & $0(0)$ & $<0.001 *$ \\
\hline Tooth extraction with osteotomy & $9(33.3)$ & $8(14.5)$ & $12(25.0)$ & 0.135 \\
\hline
\end{tabular}

(PLT: platelet count, k: 1,000 unit, <50k: PLT <50k group, 50k-100k: PLT >50k and <100k group, 100k-150k: PLT >100k and <150k group) $* P<0.05$.

Values are presented as number only, mean \pm standard deviation, or number (\%).

Sang-Hoon Kang et al: The incidence of postoperative hemorrhage after tooth extraction in patients with thrombocytopenia. J Korean Assoc Oral Maxillofac Surg 2021 
$(51.9 \%)$ received a platelet transfusion before the tooth extractions compared to the other thrombocytopenia subgroups $(P<0.001)$.

A total of 330 teeth were extracted in this study. Of the 162 maxillary teeth extracted, five exhibited postoperative bleeding complications (one anterior tooth, three premolars, and one posterior molar tooth). Six (one anterior tooth and five posterior molar teeth) of the 168 mandibular teeth showed postoperative bleeding. No significant differences in the incidence of post-extraction bleeding were found between the maxillary and mandibular teeth.(Table 3 )

The mean age of the 130 patients who underwent tooth extractions with thrombocytopenia was $63.1 \pm 15.1$ years (mean \pm standard deviation [SD]), while the mean age of the 90 patients who underwent tooth extractions with normal platelet counts was $65.7 \pm 13.7$ years (mean \pm SD) $(P=0.192)$. There was no significant difference in the mean age between the two groups. The 130 patients who underwent tooth extractions with thrombocytopenia had platelet counts of $83.6 \pm 38.1 \mathrm{k}$ (mean \pm SD). The 90 patients who underwent tooth extractions with normal platelet counts had platelet counts of $209.4 \pm 42.0 \mathrm{k}$ (mean $\pm \mathrm{SD})(P<0.001)$.

\section{Discussion}

In this study, there was a significant difference in the number of patients who had post-extraction bleeding between the thrombocytopenia group and the group with normal platelet counts.

A previous study reported that five (7.4\%) out of 200 tooth extractions in 68 patients experienced post-extraction bleeding in patients with thrombocytopenia ${ }^{5}$. Similar results were observed in our study, in which $10(7.7 \%)$ of the 130 patients who underwent a tooth extraction with thrombocytopenia ex- hibited post-extraction hemorrhagic complications, and only one $(1.1 \%)$ of the 90 patients who underwent tooth extraction with a normal platelet count had a post-extraction hemorrhagic complication.

A study reported that the risk of hemorrhagic complications following tooth extraction is high for patients with a platelet count $<40 \mathrm{k}$ and prothrombin time greater than 2.5 international normalized ratio ${ }^{6}$. In this study, patients with platelet counts $\leq 150 \mathrm{k}$ were divided into three smaller groups differing in platelet counts by $50 \mathrm{k}$. In this study, unlike the above reported results, four patients with platelet counts $<50 \mathrm{k}$, three patients with platelet counts $>50 \mathrm{k}$ and $<100 \mathrm{k}$, and three patients with platelet counts $>100 \mathrm{k}$ and $<150 \mathrm{k}$ had post-extraction hemorrhagic complications. No significant differences in the incidence of post-extraction bleeding was observed between the subgroups by platelet count within the thrombocytopenia group $(P=0.293)$. The incidence of postoperative bleeding was not related with low platelet counts between the smaller groups of the thrombocytopenia group. In cases of thrombocytopenia with platelet counts $<150 \mathrm{k}$, it was difficult to conclude that low platelet counts $(<50 \mathrm{k}, 50 \mathrm{k}-$ $100 \mathrm{k}$, and $100 \mathrm{k}-150 \mathrm{k}$ ) indicated an increased risk of postextraction hemorrhagic complications.

A systematic review on platelet counts and hemorrhagic complications following invasive dental procedures reported that platelet counts must be at least $50 \mathrm{k}$ before a surgical procedure, prophylactic platelet transfusions do not reduce the risk of postoperative hemorrhagic complications, and that local treatment on the extraction wound is important for reducing postoperative bleeding ${ }^{4}$. In our study, 18 (13.8\%) of the 130 patients who underwent tooth extractions with thrombocytopenia had received a platelet transfusion before tooth extraction, and one of these patients experienced a hemorrhagic complication. In this study, the number of patients

Table 3. Characteristics of tooth extraction according to the preoperative PLT ( $k=1,000$ unit)

\begin{tabular}{|c|c|c|c|c|}
\hline \multirow{2}{*}{ Variable } & \multicolumn{4}{|c|}{$\begin{array}{l}\text { No. of tooth extractions [post-extraction bleeding cases] } \\
\text { according to the control method of warfarin }(n=330)\end{array}$} \\
\hline & $\begin{array}{l}\text { PLT<50k } \\
(\mathrm{n}=38)[4]\end{array}$ & $\begin{array}{c}50 \mathrm{k}<\mathrm{PLT}<100 \mathrm{k} \\
(\mathrm{n}=92)[3]\end{array}$ & $\begin{array}{l}100 \mathrm{k}<\mathrm{PLT}<150 \mathrm{k} \\
\quad(\mathrm{n}=69)[3]\end{array}$ & $\begin{array}{c}150 \mathrm{k}<\mathrm{PLT}<250 \mathrm{k} \\
(\mathrm{n}=131)[1]\end{array}$ \\
\hline Maxilla $(\mathrm{n}=162)[5]$ & & & & \\
\hline Anterior:Premolar:Molar=sum & $6: 8[1]: 5=19[1]$ & $9: 17[1]: 23=49[1]$ & $7[1]: 9[1]: 16[1]=32[3]$ & $11: 16: 35=62[0]$ \\
\hline $\begin{array}{l}\text { Sim:Sim+Suture:Surgical=sum } \\
\text { Mandible }(\mathrm{n}=168)[6]\end{array}$ & $8[1]: 10: 1=19[1]$ & $33[1]: 16: 0=49[1]$ & $14: 15[2]: 3[1]=32[3]$ & $47: 13: 2=62[0]$ \\
\hline Anterior:Premolar:Molar=sum & $3[1]: 3: 13[2]=19[3]$ & $8: 13: 22[2]=43[2]$ & $5: 8: 24=37[0]$ & $11: 13: 45[1]=69[1]$ \\
\hline Sim:Sim+Suture:Surgical=sum & $6[1]: 5[2]: 8=19[3]$ & $22: 13[2]: 8=43[2]$ & $19: 9: 9=37[0]$ & $44: 12[1]: 13=69[1]$ \\
\hline
\end{tabular}

(PLT: platelet count, [ ]: post-extraction bleeding case, Anterior: central and lateral incisor, Premolar: canine and first and second premolars, Molar: first to third molars, Sim: simple extraction without primary closure, Sim+Suture: simple extraction with primary closure, Surgical: surgical extraction with/without osteotomy)

Sang-Hoon Kang et al: The incidence of postoperative hemorrhage after tooth extraction in patients with thrombocytopenia. J Korean Assoc Oral Maxillofac Surg 2021 
who received platelet transfusions before tooth extractions was too small to determine whether platelet transfusions can reduce the risk of postoperative bleeding. Preoperative platelet transfusions should be considered before invasive dental procedures under consultation with the medical department even though pre-extraction platelet transfusions do not appear to have a promising hemostatic effect during dental surgery.

This study is only about hemorrhage in patients with thrombocytopenia as they have reduced absolute platelet counts. Additional studies are necessary to compare the frequency of post-extraction bleeding in between patients with thrombocytopenia and patients who take medications affecting hemostasis. A study reported that there was no difference in the incidence of post-extraction bleeding among patients who took direct oral anticoagulants (DOACs) and warfarin, which affects hemostasis, and that $23(8.8 \%)$ of 262 patients with warfarin had hemorrhagic complications ${ }^{7}$. Our study results were similar to the abovementioned warfarin group with $10(7.7 \%)$ of the 130 patients with thrombocytopenia who underwent tooth extraction in our study experiencing post-extraction hemorrhagic complications. Therefore, the incidence of post-extraction hemorrhagic complications may be similar or less between patients who take medications that affect hemostasis such as warfarin and patients with thrombocytopenia with reduced platelet counts $(<150 \mathrm{k})^{8}$.

Rocha et al. ${ }^{9}$ reported the incidence of post-extraction bleeding where three $(4.3 \%)$ of 66 patients who underwent tooth extractions and were taking vitamin $\mathrm{K}$ antagonists exhibited postoperative hemorrhagic complications, while two $(2.9 \%)$ of 67 patients who did not take anticoagulants had postoperative hemorrhagic complications.

In our study, $10(7.7 \%)$ of the patients with thrombocytopenia had post-extraction hemorrhagic complications, suggesting that the incidence of post-extraction hemorrhagic complications can differ between patients with reduced platelet functions and those with reduced platelet counts $(<150 \mathrm{k})$. It is necessary to consider the high possibility of bleeding complications in patients with thrombocytopenia.

In our study, one $(2.3 \%)$ of the 44 patients who underwent tooth extractions involving alveolar osteotomies had post-extraction hemorrhagic complications. This indicates that a surgical extraction surgery with an alveolar osteotomy may not increase postoperative hemorrhagic complications compared to a simple extraction without an osteotomy. In addition, no significant difference in the incidence of post-extraction bleeding was observed between maxillary and mandibular teeth.
An appropriate evaluation of bleeding tendencies during tooth extractions is required even for simple extractions. In our study, 7 of the 11 patients who had hemorrhagic complications underwent suturing at the tooth extraction socket areas. Based on these results, suturing the extraction site alone does not prevent post-extraction hemorrhagic complications, and the additional use of hemostatic materials may be necessary. A randomized prospective study reported that using hemostatic materials such as hemostatic plugs can reduce the incidence of post-extraction hemorrhagic complications following tooth extractions involving only simple suturing ${ }^{10}$. Additionally, a study reported that five patients (4\%) who were administered warfarin had hemorrhagic complications following tooth extractions and were provided locally absorbable and hemostatic agents such as gelatin sponges and collagen fleeces ${ }^{11}$. Based on these results, the use of extraction techniques causing minimal wounds, hemostatic materials, and appropriate hemostatic measures can prevent postextraction hemorrhagic complications.

Local treatment is important to reduce the risk of postextraction hemorrhagic complications in patients with thrombocytopenia. However, simply performing an atraumatic extraction in addition to applying hemostatic materials to the extraction socket is not sufficient as a local treatment. Unnecessary suturing simply causes a wound as the needle punctures the extraction site. While applying hemostatic materials during suturing may be useful, more scientific evidence is needed to support their use in all patients since no study has demonstrated that using hemostatic materials is beneficial for all tooth extraction procedures with thrombocytopenia patients. Therefore, it is necessary to predict the risk of bleeding through an evaluation of the extraction site and choose an appropriate treatment approach for bleeding control. Studies have reported that an appropriate evaluation of the hemorrhagic nature of the tooth extraction site is necessary for patients with thrombocytopenia to reduce post-extraction bleeding ${ }^{4,5,12}$. In addition, high levels of experience and care of the surgeon are required for preventing postoperative hemorrhagic complications ${ }^{4,5,12}$.

Studies assessing the severity of post-extraction bleeding are rare. One study graded the severity of post-extraction bleeding in patients with thrombocytopenia ${ }^{12}$. In this previous study, the severity of bleeding was graded from 1 to 3 . Grade 1 bleeding can be stopped by simple compression, while grade 2 bleeding requires additional measures such as the use of a hemostat, re-suturing, or surgical splints ${ }^{12}$. Grade 3 bleeding requires vitamin $\mathrm{K}$, fresh frozen plasma, or clotting factor 
replacement transfusions. During tooth extractions, bleeding may be graded into one that can be stopped through compression with wet gauze, one that requires additional sutures and hemostatic materials, and one that requires additional platelet transfusions ${ }^{12}$. Considering that most post-extraction hemorrhagic complications in patients with thrombocytopenia were treated by local treatments, grade 2 bleeding may be the most common. In our study, a hemorrhagic complication occurred at a single location even in patients who had multiple teeth removed. This suggests that while it is important to consider the patient's systemic conditions, local treatment including evaluation and bleeding control applied at the tooth extraction site may also be important.

A surgeon must stop the bleeding at the tooth extraction site through compression, sutures, and the use of hemostatic materials during tooth extraction procedures. Patients should be discharged after hemostasis confirmation has been achieved in order to reduce the incidence of postoperative hemorrhagic complications.

Tooth extractions are performed on patients with serious systemic conditions such as thrombocytopenia. Based on previous findings and the findings from this study, the risk of post-extraction hemorrhagic complications is higher in patients with thrombocytopenia, and local treatment including evaluation and bleeding control applied at the tooth extraction site is more important than whether the patient received platelet transfusions or the patient's absolute platelet count. It is necessary to assess the severity of immediate post-extraction bleeding on tooth extraction sites in patients with thrombocytopenia and appropriate measures taken before patients are to be discharged.

\section{Conclusion}

Surgeons must perform an atraumatic operation and assess bleeding patterns during tooth extractions to choose an appropriate hemostatic measure to prevent post-extraction bleeding in patients with thrombocytopenia (platelet count $<150 \mathrm{k}$ ). They must also consider using sutures and local hemostatic agents at the extraction site. In cases of thrombocytopenia with platelet counts $<150 \mathrm{k}$, it is difficult to conclude that low platelet counts (e.g., $<50 \mathrm{k}, 50 \mathrm{k}-100 \mathrm{k}, 100 \mathrm{k}-150 \mathrm{k}$ ) indicate an increased risk of post-extraction hemorrhagic complications.

\section{ORCID}

Sang-Hoon Kang, https://orcid.org/0000-0003-3335-3040

Min-Jun Kang, https://orcid.org/0000-0002-8004-3689

\section{Authors' Contributions}

M.J.K. and S.H.K. obtained data and wrote the manuscript. S.H.K. and M.J.K. drafted the manuscript. S.H.K. and M.J.K. participated in article design and coordination and carefully reviewed and revised the manuscript. All the authors read and approved the final manuscript.

\section{Acknowledgements}

This work was supported by the Clinical Research Fund of the National Health Insurance Service Ilsan Hospital (NHIMC2020CR084).

\section{Ethics Approval and Consent to Participate}

This retrospective study was approved by the institutional review board of the National Health Insurance Service Ilsan Hospital (No. NHIMC 2020-09-004-001). Because of the retrospective nature of this study, which did not use identifying personal information, the institutional review board of the hospital exempted the study from the requirement to obtain written informed consent from the study subjects.

\section{Conflict of Interest}

No potential conflict of interest relevant to this article was reported.

\section{References}

1. Akhlaghi F, Khaheshi I, Amirhassani S, Tabrizi R. Do antiplatelet drugs increase the risk of bleeding after tooth extraction? A casecrossover study. Int J Oral Maxillofac Surg 2017;46:1475-8. https:// doi.org/10.1016/j.ijom.2017.06.002

2. Sadeghi-Ghahrody M, Yousefi-Malekshah SH, Karimi-Sari H, Yazdanpanah H, Rezaee-Zavareh MS, Yavarahmadi M. Bleeding after tooth extraction in patients taking aspirin and clopidogrel $\left(\right.$ Plavix $\left.^{\circledR}\right)$ compared with healthy controls. Br J Oral Maxillofac Surg 2016;54:568-72. https://doi.org/10.1016/j.bjoms.2016.02.036

3. Bajkin BV, Vujkov SB, Milekic BR, Vuckovic BA. Risk factors for bleeding after oral surgery in patients who continued using oral anticoagulant therapy. J Am Dent Assoc 2015;146:375-81. https:// doi.org/10.1016/j.adaj.2015.01.017

4. Karasneh J, Christoforou J, Walker JS, Manfredi M, Dave B, Diz Dios P, et al. World Workshop on Oral Medicine VII: platelet count 
and platelet transfusion for invasive dental procedures in thrombocytopenic patients: a systematic review. Oral Dis 2019;25 Suppl 1:174-81. https://doi.org/10.1111/odi.13082

5. Fillmore WJ, Leavitt BD, Arce K. Dental extraction in the thrombocytopenic patient is safe and complications are easily managed. J Oral Maxillofac Surg 2013;71:1647-52. https://doi.org/10.1016/ j.joms.2013.05.011

6. Cocero N, Bezzi M, Martini S, Carossa S. Oral surgical treatment of patients with chronic liver disease: assessments of bleeding and its relationship with thrombocytopenia and blood coagulation parameters. J Oral Maxillofac Surg 2017;75:28-34. https://doi. org/10.1016/j.joms.2016.08.033

7. Yoshikawa H, Yoshida M, Yasaka M, Yoshida H, Murasato Y, Fukunaga D, et al. Safety of tooth extraction in patients receiving direct oral anticoagulant treatment versus warfarin: a prospective observation study. Int J Oral Maxillofac Surg 2019;48:1102-8. https://doi.org/10.1016/j.ijom.2019.01.013

8. Lee JS, Kim MK, Kang SH. Effect of warfarin discontinuation on the incidence of postoperative bleeding in tooth extraction. $\mathrm{J}$ Korean Assoc Oral Maxillofac Surg 2020;46:228-34. https://doi. org/10.5125/jkaoms.2020.46.4.228

9. Rocha AL, Oliveira SR, Souza AF, Travassos DV, Abreu LG, Ribeiro DD, et al. Bleeding assessment in oral surgery: a cohort study comparing individuals on anticoagulant therapy and a nonanticoagulated group. J Craniomaxillofac Surg 2019;47:798-804. https://doi.org/10.1016/j.jcms.2019.01.049
10. Giudice A, Esposito M, Bennardo F, Brancaccio Y, Buti J, Fortunato L. Dental extractions for patients on oral antiplatelet: a withinperson randomised controlled trial comparing haemostatic plugs, advanced-platelet-rich fibrin (A-PRF+) plugs, leukocyte- and platelet-rich fibrin (L-PRF) plugs and suturing alone. Int J Oral Implantol (Berl) 2019;12:77-87.

11. Svensson R, Hallmer F, Englesson CS, Svensson PJ, Becktor JP. Treatment with local hemostatic agents and primary closure after tooth extraction in warfarin treated patients. Swed Dent J 2013;37:71-7.

12. Hasegawa T, Yanamoto S, Tachibana A, Kojima Y, Koyama Y, Maeda M, et al.; Japanese Study Group of Cooperative Dentistry with Medicine (JCDM). The risk factors associated with postoperative hemorrhage after tooth extraction: a multi-center retrospective study of patients receiving oral antithrombotic therapy. Oral Maxillofac Surg 2017;21:397-404. https://doi.org/10.1007/s10006-017$0645-\mathrm{y}$

How to cite this article: Kang SH, Kang MJ. The incidence of postoperative hemorrhage after tooth extraction in patients with thrombocytopenia. J Korean Assoc Oral Maxillofac Surg 2021;47:190-196. https://doi.org/10.5125/jkaoms.2021.47.3.190 